\title{
OPERATION-LEVEL 4D MODELING AND VISUALIZATION FOR MODULAR BUILDING BASED ON STANDARD ACTIVITY LIBRARY
}

\author{
Hengwei Wang ${ }^{1}$, Jiarui Lin $^{2}$, and Jianping Zhang ${ }^{3}$
}

\begin{abstract}
Modular or prefabricated building plays an important role in lean construction and green building. Building information modeling and 4D bring a new paradigm for data modeling and visualization in the Architecture, Engineering and Construction domain. Meanwhile, lots of time was consumed when modeling and visualizing the dynamic construction process based on $4 \mathrm{D}$. It takes more time to create an operation-level 4D model for technical specification, that always adopted to ensure the quality and safety. Given that modular or prefabricated building projects have lots of similar construction activities, this paper proposed a 4D modeling and visualization approach for modular building, which can persist generalized construction activities and operations in standard activity library and fully utilize them in schedule detailing and visualization. The approach is designed and applied based on previous researches and applications of 4D BIM and has been used in a practical project of modular residential project to illustrate the feasibility of the work and the possibility of future research.
\end{abstract}

Keywords: 4D modeling, operation level, Building Information Model, modular building, prefabricated building.

\section{INTRODUCTION}

Building Information Modeling and 4D bring a new paradigm for data modeling and visualization in the Architecture, Engineering and Construction domain, and has been applied to help the management of schedule, resource, safety and site (Shang and Shen 2016, Kang et al. 2016, Zhou et al. 2015). Meanwhile, lots of time was consumed when modeling and visualizing the dynamic construction activities based on 4D.

Technical specification is an important task to tell the procedures and the key points to workers to prevent the mistake or fault in construction. The traditional way to describe the activities in the technical specification is to use graphs and texts. But it is difficult for workers to understand. Therefore, 3D animations produced manually are applied. However, in many cases, the contractors are not willing to pay for such works. The operation-level 4D model provides a more automatic way for generating 3D animations (Zhang et al. 2014, Han et al. 2014, Han et al. 2012, Kamat et al. 2010), while it takes time to create such a model.

Modular or prefabricated building plays an important role in lean construction and green building. The construction activities of each of the limited types of building elements

PhD Candidate, Department of Civil Engineering, Tsinghua University, Beijing, China, whw13@mails.tsinghua.edu.cn

2 Postdoctoral Researcher, Department of Civil Engineering, Tsinghua University, Beijing, China, jiarui_lin@foxmail.com

3 Professor, Department of Civil Engineering, Tsinghua University, Beijing, China, zhangjp@tsinghua.edu.cn 
are similar in a modular or prefabricated building project. It implies that when the contractor has several standard activities of the project, 4D BIM can be detailed to operation-level in an effective way.

The purpose of this study is to generate substantial activity descriptions and visualizations in technical specifications as simply as possible. Considering that many construction activities of modular or prefabricated buildings have the same operations and operation sequences, an approach consist of data model and workflow for 4D modeling and visualization of modular or prefabricated building projects is proposed. By implementing and demonstrating the method, the feasibility and flexibility of the proposed method as well as future improvement are concluded and discussed at the end of this paper.

\section{METHODOLOGY}

\subsection{Basic Framework}

The basic framework of the method contains two main parts, the data model and the workflow. The data model is shown in Figure 1.

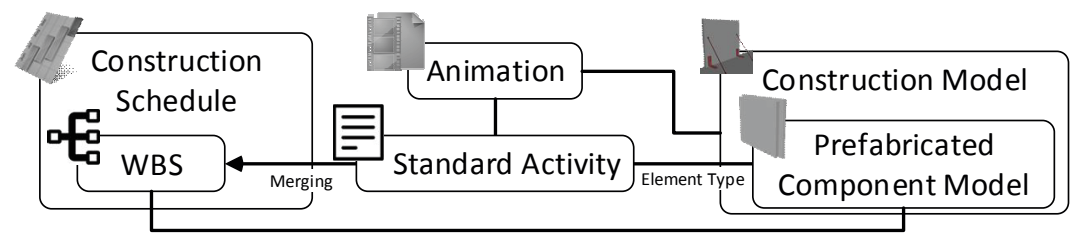

Figure 1: Data model

The core of the data model is the standard activity. The animation is related to standard activity directly, and the construction model, which contains the model of prefabricated component and other temporal elements, is also associated with the animation through element type. Thus, WBS and schedule of the whole construction process can be detailed by incorporating pre-defined standard activities. And the relationship can be established semi-automatically with the relationship between type of the prefabricated component and its corresponding task in WBS. The workflow is shown in Figure 2.

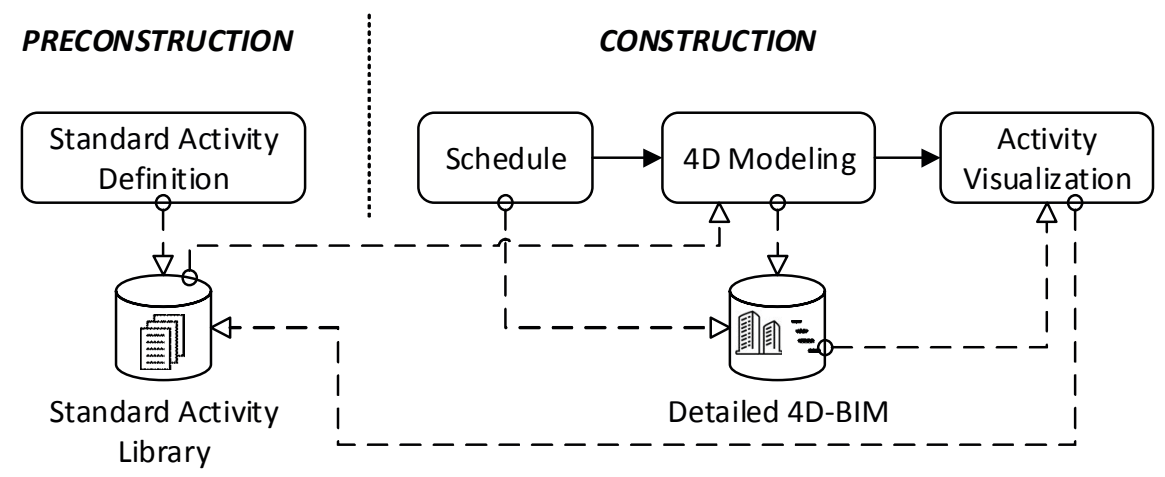

Figure 2: Workflow for 4D Modeling and Visualization

In the workflow for 4D modeling and visualization, a standard activity library was set up before modeling and visualization of the construction process. The standard activity library contains lots of pre-defined standard activities for the construction of modular building. Each standard activity is corresponding to one type of standard building elements and describes different steps and their sequence for the construction or installation of the 
element. Every step has animations to represent the durations and actions of related elements.

In the construction phase, works are based on the detailed 4D BIM. The whole workflow consists of three main activities:

1. Scheduling. This is the basic job of schedule managers. They always create the WBS (Work Breakdown Structure) and determine the time point of the works.

2. 4D Modeling. Associating the scheduling result with BIM and integrating the schedule with standard activities by the WBS, we can get the detailed 4D BIM.

3. Activity Visualization. Based on the detailed 4D BIM, we can visualize the activity in operation level automatically, and put the animation result back into the standard activity library.

\subsection{Standard Activity Library}

A standard activity library consists of several standard activities. The structure of a standard activity is shown in Figure 3.

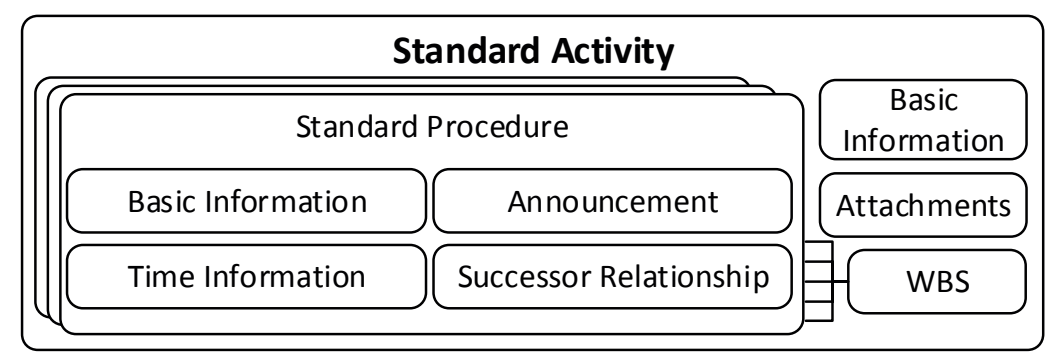

Figure 3: Standard activity

Each standard activity contains several standard procedures, such as making level, lofting, lifting, etc. A standard activity is considered as a workflow in general. However, it is difficult to merge a workflow into the construction schedule. Therefore, we choose to break down the standard activity. The model of the standard activity contains the basic information, attachment and the WBS which is comprised of standard procedures.

\section{IMPLEMENTATION}

\subsection{Animation Creation}

Animations are created for the procedures in standard activities. Generally, all animations in construction simulation can be decomposed into a set of basic animation components, which are listed in Table 1.

The types of animation components are classified into three kinds: the matrix transformation, the transparency and the parameter. To place, to translate or to rotate is to change the spatial state of a 3D model by modify the transform matrix. The appearance or disappearance of a 3D model is a change process of transparency. Some complicated animations, which can be determined by unique parameters, such as hoisting, should be pre-defined in unique construction elements. 
Table 1: Basic animation components

\begin{tabular}{cc}
\hline Type & Arguments \\
\hline Place & Position \\
Translate & Translation vector \\
Rotate & Rotation axes, rotation angle \\
Appear & - \\
Disappear & - \\
\hline
\end{tabular}

\subsection{Schedule Detailing based on Standard Activity}

Basic structure for scheduling detailing is the WBS. The relationship between the whole WBS and standard activity can be established automatically by related building element. A typical example of generating the detailed schedule is shown in Figure 4. In 4D BIM, the work is ordinarily broken down by spatial structure, and the finest node is the construction segment. One segment may be related with several building elements, which including the prefabricated component in modular building projects. For example, if the first construction segment of the walls in a storey is related to a wall, the standard activity for the installation of the wall can be merged into the whole WBS by connecting with the segment node. In this way, the WBS is extended and we can get a more detailed schedule.

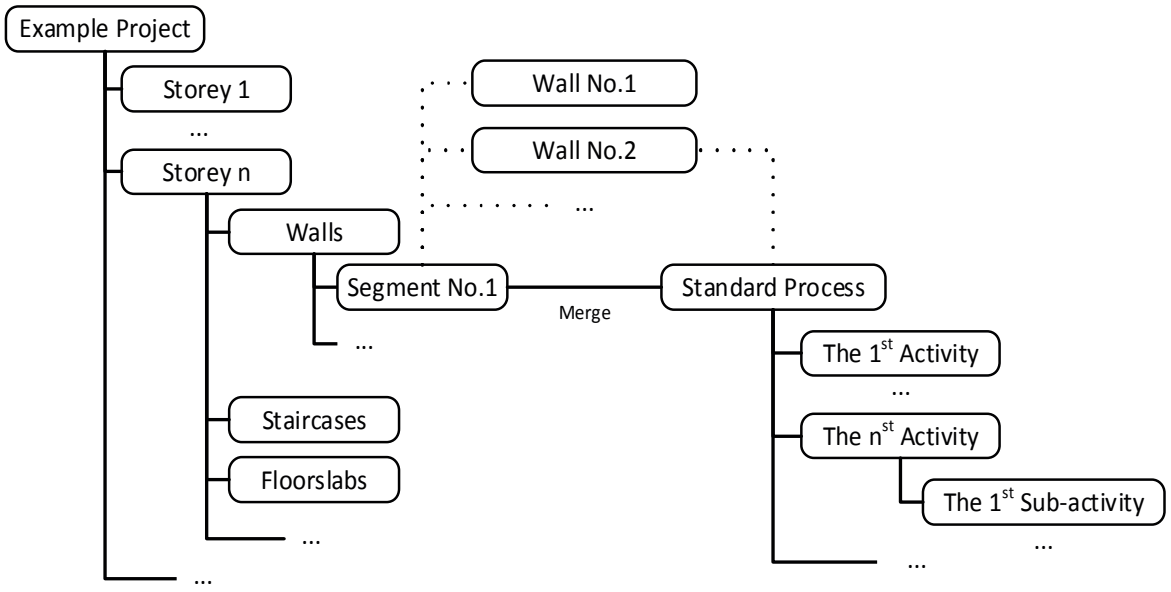

Figure 4: Typical example of generating the detailed schedule

\subsection{Visualization}

The activity visualization is based on the detailed 4D BIM with animations. The procedure has two steps:

1. 4D simulation to determine the construction status in the certain day of the activity.

2. Visualizing the activity based on the construction status.

The first step is based on traditional 4D BIM. And the second step is the emphasis. In this step, the result of activity visualization should be pre-computed in order to insure the usability. Therefore, the key-frames should be pre-generated. Considering the actual requirement, we choose one second as a minimum interval of the key-frames. The two main steps of pre-generating the key-frames is shown below. 
1. Looping over all of the animation components, calculating the variations between included adjacent key-frames.

2. Calculate the 3D status of the key-frames based on the variations got in step one from the start to the end of the activity.

The time complexity of the algorithm above is $\mathrm{O}\left(\left(N_{a}+N_{k} \overline{N_{a}}\right) \overline{N_{e}}\right)$. Among the formula, $N_{a}$ is the total amount of animation components; $N_{k}$ is the total amount of key-frames; $\overline{N_{a}}$ is the average amount of animation components refer to one key-frame; and $\overline{N_{e}}$ is the average amount of the construction elements related to one animation component. The duration of an activity is hardly able to be longer than a day, which is 86400 seconds. Hence, it is acceptable to consider 86400 as the maximum value of $N_{k}$. Moreover, $\overline{N_{a}} \leq$ $N_{a}$ and $\overline{N_{e}}$ is always in single digitals. Supposing $\overline{N_{a}}=N_{a}$ and $\overline{N_{e}}=1$, the time complexity approximates to $O\left(N_{a} N_{k}\right) \leq O\left(86400 N_{a}\right)$, which is acceptable for precomputing of activity visualization.

\section{CASE STUDY}

\subsection{Application Strategy}

The method is applied based on a 4D BIM application which is developed by the 4D-BIM group in the department of civil engineering of Tsinghua University and is able to support scheduling, BIM import and 4D BIM modelling. All the data, which include the standard activity, the whole schedule and the BIM, are from a modular residential project in Hefei, China. The standard activities and their corresponding animations are defined based on experience of engineers.

\subsection{Standard activity}

A typical standard activity of the project is shown in Figure 5.
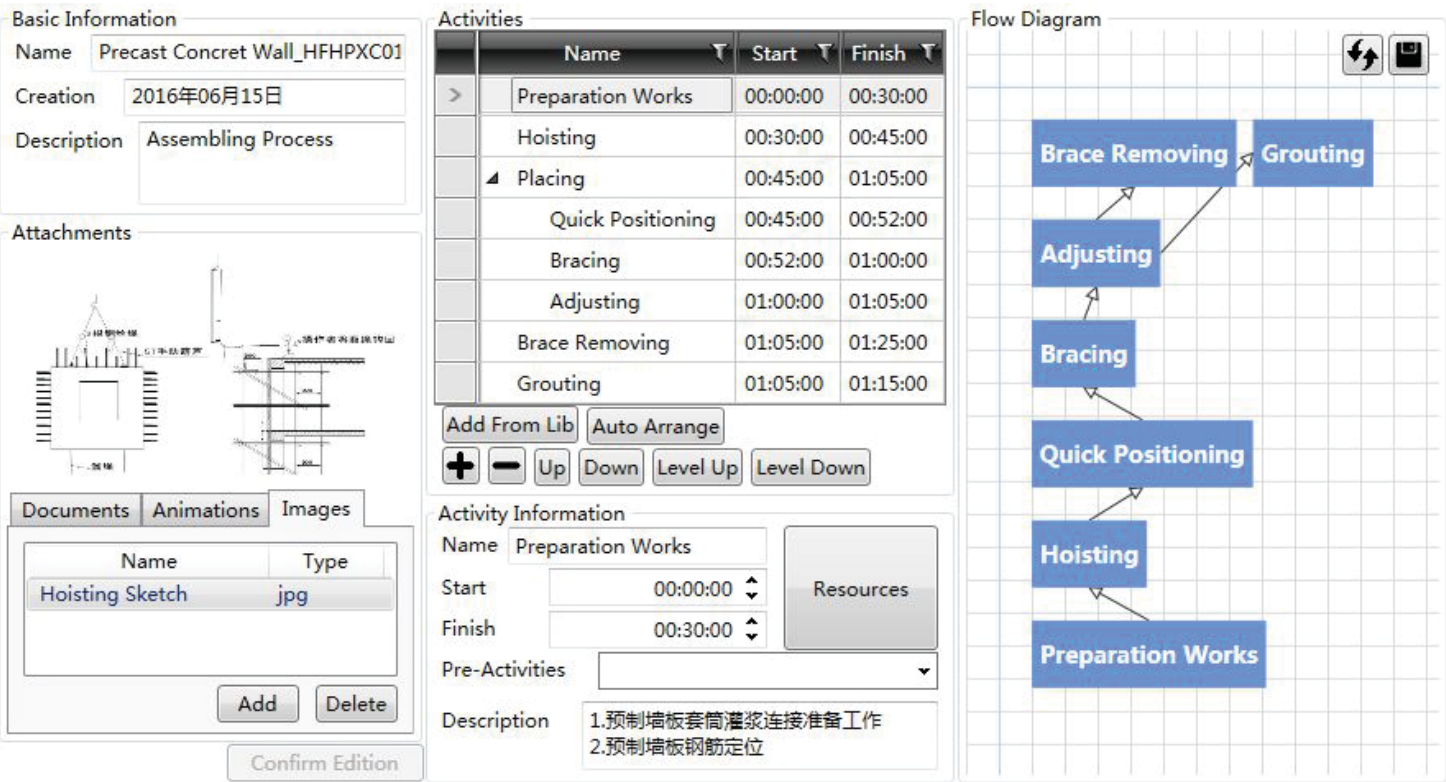

Figure 5: Standard activity of precast concrete wall assembling

The standard assembling activity of precast concrete wall contains five main procedures: preparation works, hoisting, placing, brace removing and grouting. And placing procedure 
has three sub procedures: quick positioning, bracing and adjusting. The entire workflow is existed based on the precedence relationship between these procedures.

\subsection{Animation Definition}

The animation of a procedure is defined before the pre-computation of the visualization. The scripts in a hoisting procedure is shown in Figure 6. As an example, the script of object lifting is shown in Figure 7. Apart from the necessary information, we use Bezier Curve to improve the visualization results.

\begin{tabular}{|l|l|l|l|l|l|}
\hline Hame & Type & Start & Finish & Related Element Type & Related Element ID \\
\hline Object lifting & Rotation & $08: 30: 00$ & $08: 31: 00$ & B & 2423 \\
\hline Lifting when hoisting & Hoisting & $08: 30: 00$ & $08: 31: 00$ & S & 1 \\
\hline Object lifting & Translation & $08: 31: 00$ & $08: 42: 00$ & B & 2423 \\
\hline Lifting when hoisting & Hoisting & $08: 31: 00$ & $08: 42: 00$ & S & 1 \\
\hline Object translating & Translation & $08: 42: 00$ & $08: 45: 00$ & B & 2423 \\
\hline Translating when hoisting & Hoisting & $08: 42: 00$ & $08: 45: 00$ & S & 1 \\
\hline
\end{tabular}

Figure 6: Animation scripts of hoisting procedure

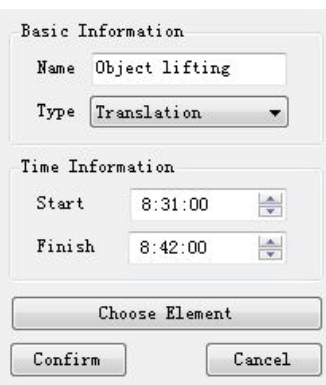

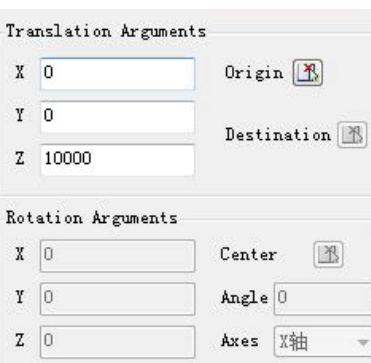
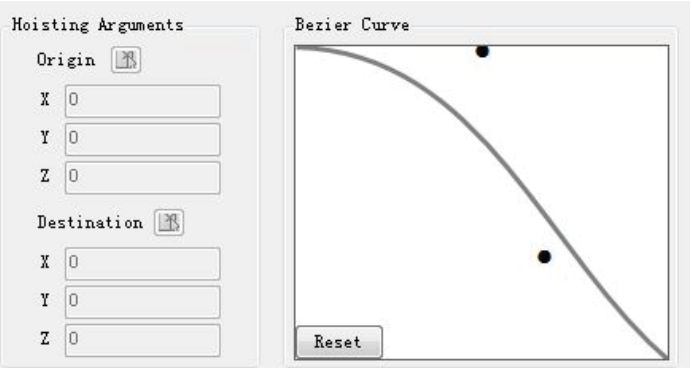

Figure 7: Script of object lifting

\subsection{Schedule Detailing}

The structure of the detailed schedule is shown in Figure 8.

\begin{tabular}{|c|c|c|c|c|c|c|c|c|c|}
\hline & & & & \multicolumn{3}{|c|}{ Activity Name } & \multirow{2}{*}{$\begin{array}{c}\text { Animation } \\
\text { s.s. }\end{array}$} & Start & \multirow{2}{*}{$\begin{array}{l}\text { Finish } \\
8: 30: 00\end{array}$} \\
\hline & & & & , & \multicolumn{2}{|c|}{ Preparation Works } & & $8: 00: 00$ & \\
\hline & & & & & \multicolumn{2}{|l|}{ Hoisting } & 㫮 & $8: 30: 00$ & 8:45:00 \\
\hline & & & & & \multicolumn{2}{|c|}{ Placing } & 픔 & $8: 45: 00$ & 9:05:00 \\
\hline & & & & & \multicolumn{2}{|c|}{ Quick Positioning } & 兴 & $8: 45: 00$ & 8:52:00 \\
\hline \multicolumn{2}{|l|}{ Task Name } & Schedule Start & Schedule Finish & & \multicolumn{2}{|c|}{ Bracing } & 临 & $8: 52: 00$ & 9:00:00 \\
\hline \multicolumn{2}{|c|}{ - $\boxminus \diamond$ HFHPXC Project } & 2016-05-27 & 2016-10-17 & & \multicolumn{2}{|l|}{ Adjusting } & 党 & 9:00:00 & 9:05:00 \\
\hline$-\square 84 C$ & -Structure & $2016-05-27$ & $2016-10-17$ & & \multicolumn{2}{|l|}{ Brace Removing } & 임 & 9:05:00 & 9:25:00 \\
\hline , $\square$ & Underground & $2016-05-27$ & 2016-06-01 & & \multicolumn{2}{|l|}{ Grouting } & - & 9:05:00 & 9:15:00 \\
\hline$\square 81$ & $1 \mathrm{~F}$ & 2016-06-02 & 2016-06-07 & & \multicolumn{2}{|c|}{ Preperation Works } & 무 & 9:05:00 & 9:35:00 \\
\hline 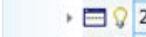 & $2 \mathrm{~F}$ & 2016-06-08 & 2016-06-13 & & \multicolumn{2}{|c|}{ Hoisting } & 嗂 & 9:35:00 & 9:50:00 \\
\hline , $\square 83$ & $3 F$ & 2016-06-14 & 2016-06-19 & & \multicolumn{2}{|c|}{ Placing } & 㫮 & 9:50:00 & $10: 10: 00$ \\
\hline,$\square 84$ & $4 \mathrm{~F}$ & $2016-06-20$ & $2016-06-25$ & & \multicolumn{2}{|c|}{ Quick Positioning } & 㫮 & 9:50:00 & 9:57:00 \\
\hline . 885 & $5 F$ & 2016-06-26 & 2016-07-01 & & \multicolumn{2}{|c|}{ Bracing } & 믐 & $9: 57: 00$ & 10:05:00 \\
\hline . $\square 86$ & $6 F$ & 2016-07-02 & 2016-07-07 & & \multicolumn{2}{|l|}{ Adjusting } & 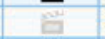 & 10:05:00 & $10: 10: 00$ \\
\hline$-\square 07$ & $7 F$ & $2016-07-08$ & 2016-07-13 & & \multicolumn{2}{|l|}{ Brace Removing } & 菖 & 10:10:00 & 10:30:00 \\
\hline \multicolumn{2}{|c|}{ - $\boxminus \nabla$ Slabs } & 2016-07-08 & 2016-07-09 & & \multirow{2}{*}{\multicolumn{2}{|c|}{ Girnutinn }} & 세 & $1 n \cdot 1 n \cdot n n$ & $1 n \cdot 3 n \cdot n n$ \\
\hline & 돔 Segment 1 & 2016-07-08 & 2016-07-08 & & & & & & $s^{10 \cdot 3 n \cdot n n}$ \\
\hline & 놈 2 Segment 2 & 2016-07-09 & 2016-07-09 & \multirow{2}{*}{\multicolumn{3}{|c|}{ Pre-Compute Add Process }} & Result to & 0 Lb & Lb Edit \\
\hline \multicolumn{2}{|c|}{ - $巴 8$ Precast Wals } & 2016-07-11 & $2016-07-12$ & & \multirow{2}{*}{\multicolumn{3}{|c|}{ Is Simulation Process Name }} & \multirow{2}{*}{\multicolumn{2}{|c|}{ Duration }} \\
\hline & Segment 1 & $2016-07-11$ & 2016-07-11 & & & & & & \\
\hline & 동 Segment 2 & 2016-07-12 & 2016-07-12 & \multirow[t]{2}{*}{. } & 口 & Two Wals A & isembing $\mathrm{N}$.. & .. 02:30:00 & \\
\hline$-\square$ & 8 Precast Staircases & 2016-07-13 & 2016-07-13 & & $\square$ & General Wal & Constructio.. & ... $07: 00: 00$ & \\
\hline & 힘일 Segment & $2016-07-13$ & 2016-07-13 & & & & & & \\
\hline$\square 88$ & $8 \mathrm{~F}$ & 2016-07-14 & 2016-07-19 & & & & & & \\
\hline$\boxminus 89$ & $9 F$ & 2016-07-20 & $2016-07-25$ & & & & & & \\
\hline $\bar{\square} \cap \overline{1}$ & ine & $3016 \cap 736$ & 2016 ח7 21 & & & & & & \\
\hline
\end{tabular}

Figure 8: Detailed schedule 
Each construction segment contains the works of a day. For example, the works of walls in the seventh floor are divided into two segments. We correlate a standard activity of two walls assembling to the first segment. The activity starts at 8 a.m. and finish at 10:20 a.m.

\subsection{Visualization Result}

The assembly activity of two walls on the seventh floor is visualized automatically based on the prepared data and the result is shown in Figure 9.
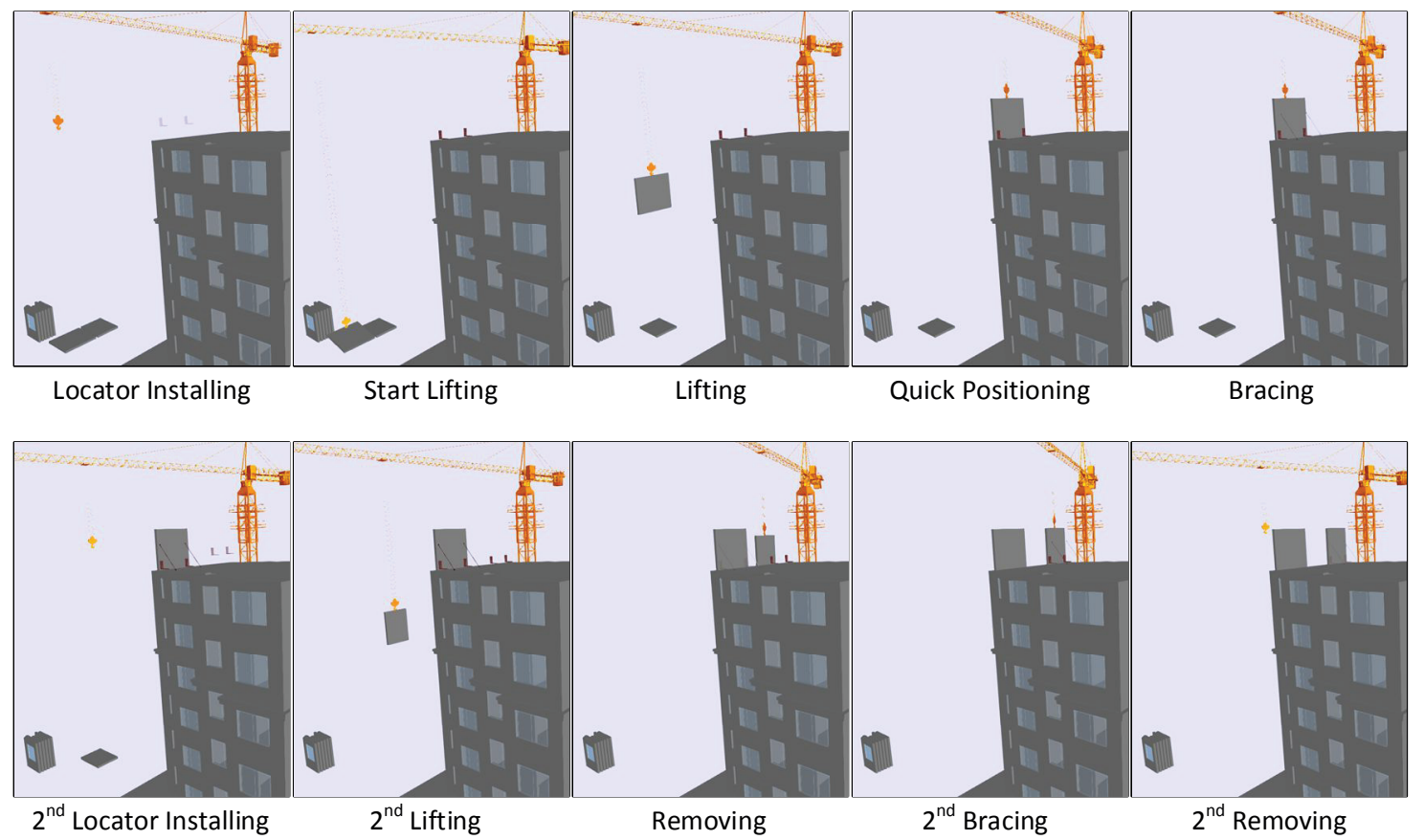

Figure 9: Visualization result

\section{CONCLUSION AND DiscUSSION}

In this study, an approach based standard activity library for 4D modeling and visualization is proposed, which can be used in construction visualization and management of modular buildings. According to the results of the case study, it is illustrated that the method proposed for $4 \mathrm{D}$ modeling and visualization for modular building projects is feasible. Concretely, five points are emphasized as follows:

- Standard activity provides a new way for modeling and persisting construction information and knowledge.

- The structure of the standard activity in this study is feasible and flexible for further utilization.

- Detailed schedule can be generated based on the standard activity.

- The method and the algorithm for activity visualization based on detailed 4D BIM in this study are highly automatic with complete data.

- Framework proposed in this paper can eliminate rework and reduce time consumed in schedule detailing and visualization.

However, several problems and key points still need further research and extension: 
- Appending resource information to standard activity for resource management in construction.

- Finding a more effective method to establish the relationship between the whole WBS and the standard activity.

- Designing a fully integrated schedule structure, rather than only attaching the standard activities to WBS.

- Automatic animation creation.

- Model and method improvement that makes it possible to support different construction or installation methods of building elements with the same type.

- Multi-LOD support of schedule.

- Supporting visualization of long-term activity.

\section{ACKNOWLEDGMENTS}

We are grateful for the support provided by the National Natural Science Foundation of China (No.51278274), the National High-tech Research and Development Program of China (No.2013AA041307), and the Tsinghua University-Glodon Joint Research Center for Building Information Model (RCBIM).

\section{REFERENCES}

Han, S. H., Hasan, S., Bouferguène, A., Al-Hussein, M., and Kosa, J. (2014). Utilization of $3 \mathrm{D}$ visualization of mobile crane operations for modular construction on-site assembly. Journal of Management in Engineering, 31(5), 04014080.

Han, S. H., Al-Hussein, M., Al-Jibouri, S., and Yu, H. (2012). Automated post-simulation visualization of modular building production assembly line. Automation in construction, 21, 229-236.

Kang, L. S., Kim, H. S., Moon, H. S., and Kim, S. K. (2016). Managing construction schedule by telepresence: Integration of site video feed with an active $\mathrm{nD}$ CAD simulation. Automation in Construction, 68, 32-43.

Kamat, V. R., Martinez, J. C., Fischer, M., Golparvar-Fard, M., Peña-Mora, F., and Savarese, S. (2010). Research in visualization techniques for field construction. Journal of construction engineering and management, 137(10), 853-862.

Shang, Z., and Shen, Z. A Framework for a Site Safety Assessment Model Using Statistical 4D BIM-Based Spatial-Temporal Collision Detection. In Construction Research Congress 2016 (pp. 2187-2196).

Zhou, Y., Ding, L., Wang, X., Truijens, M., and Luo, H. (2015). Applicability of 4D modeling for resource allocation in mega liquefied natural gas plant construction. Automation in construction, 50, 50-63.

Zhang, J., Yu, F., Li, D., and Hu, Z. (2014). Development and Implementation of an Industry Foundation Classes-Based Graphic Information Model for Virtual Construction. Computer-Aided Civil and Infrastructure Engineering, 29(1), 60-74. 